\title{
Unravelling enzymatic discoloration in potato through a combined approach of candidate genes, QTL, and expression analysis
}

\author{
Jeroen S. Werij • Bjorn Kloosterman • Carolina Celis-Gamboa • C. H. Ric de Vos • \\ Twan America · Richard G. F. Visser · Christian W. B. Bachem
}

Received: 11 December 2006 / Accepted: 14 April 2007 / Published online: 11 May 2007

(C) Springer-Verlag 2007

\begin{abstract}
Enzymatic discoloration (ED) of potato tubers was investigated in an attempt to unravel the underlying genetic factors. Both enzyme and substrate concentration have been reported to influence the degree of discoloration and as such this trait can be regarded as polygenic. The diploid mapping population $\mathrm{C} \times \mathrm{E}$, consisting of 249 individuals, was assayed for the degree of ED and levels of chlorogenic acid and tyrosine. Using this data, Quantitative Trait Locus (QTL) analysis was performed. Three QTLs for ED have been found on parental chromosomes C3, C8, E1, and E8. For chlorogenic acid a QTL has been identified on $\mathrm{C} 2$ and for tyrosine levels, a QTL has been detected on C8. None of the QTLs overlap, indicating the absence of genetic correlations between these components underlying ED, in contrast to earlier reports in literature. An obvious candidate gene for the QTL for ED on Chromosome 8 is polyphenol oxidase (PPO), which was previously mapped on chromosome 8 . With gene-specific primers for PPO gene POT32 a CAPS marker was developed. Three different alleles (POT32-1, -2,
\end{abstract}

Communicated by R. Waugh.

J. S. Werij $(\bowtie) \cdot$ B. Kloosterman · C. Celis-Gamboa ·

R. G. F. Visser · C. W. B. Bachem

Graduate School Experimental Plant Sciences,

Laboratory of Plant Breeding, Wageningen University

and Research Centre, PO Box 386,

6700 AJ Wageningen, The Netherlands

e-mail: jeroen.werij@wur.nl

J. S. Werij · C. H. R. de Vos · T. America ·

R. G. F. Visser - C. W. B. Bachem

Centre for BioSystems Genomics,

Wageningen, The Netherlands

C. H. R. de Vos - T. America

Plant Research International,

Wageningen, The Netherlands and -3) could be discriminated. The segregating POT32 alleles were used to map the POT32 CAPS marker and QTL analysis was redone, showing that POT32 coincides with the QTL peak. A clear correlation between allele combinations and degree of discoloration was observed. In addition, analysis of POT32 gene expression in a subset of genotypes indicated a correlation between the level of gene expression and allele composition. On average, genotypes having two copies of allele 1 had both the highest degree of discoloration as well as the highest level of POT32 gene expression.

\section{Introduction}

Enzymatic discoloration (ED) in potato tubers is the process in which phenolic compounds are oxidized by the enzyme polyphenol oxidase (PPO) to quinones after which the quinones are transformed to dark pigments (melanins) (Friedman 1997). This discoloration results in considerable economic losses for the food processing and retail industry. Prevention of enzymatic browning in food can be achieved by the use of sulfiting agents; however, in recent years doubt has arisen about the safety of sulfites for human consumption. An environmentally and nutritionally safe approach would be to breed new potato cultivars which do not display ED. Breeding for ED resistant potatoes first requires a thorough understanding of the genetic factors underlying the trait. Until now, research has been done to dissect the underlying mechanisms of the trait. In general two approaches were taken, either focussing on the enzyme PPO or on the substrate of the enzymatic reaction. From this research, PPO has been reported to be one of the main causes for ED (Vaughn et al. 1988). When the PPO gene expression was silenced, the transformed lines showed a significantly reduced discoloration reaction (Bachem et al. 
1994). PPO genes in potato comprise a genetically linked gene family of at least six genes, namely POTP1 and POTP2 (Hunt et al. 1993) and POT32, POT33, POT41, and POT72 (Thygesen et al. 1995). The genes are differentially expressed, with POT32 being the major form expressed in tubers besides POT33 and POT72. POTP1 and POTP2 are mainly expressed in leaves and flowers (Thygesen et al. 1995). This tissue-specific expression of PPO genes was also demonstrated by Newman et al. (1993) in tomato. The tomato PPO genes share greater sequence similarity with their orthologs in potato than with their paralogous PPO genes in tomato. POT32 has been shown to be the cause of browning in Solanum hjertingii, where a truncated version of the gene prevents ED (Culley et al. 2000).

Besides the enzymatic aspects of PPO on ED, there is also a substrate aspect to the trait. The main substrate consists of phenolic compounds. As mentioned, PPO oxidizes phenolic compounds to melanins and $90 \%$ of the total phenolic content of potato tubers consists of chlorogenic acid although no correlation was observed between chlorogenic acid and discoloration (Friedman 1997). A high correlation between tyrosine content and the results of the abrasive peel test, comparable with ED, was detected (Corsini et al. 1992). Another important determinant is the partitioning of tyrosine between tuber protein and the free amino acid pool, where high free amino acid levels corresponded with a high level of discoloration (Corsini et al. 1992). However, other research reported that in spite of an observed high correlation between free tyrosine and discoloration, the amount of tyrosine does not seem to be the determining factor for ED (Mondy and Munshi 1993).

Based on the hypothesis that multiple factors, at least enzyme and substrate, are involved in determining ED we can regard the trait ED as being a quantitative trait. Quantitative Trait Locus (QTL) analysis (Lander and Botstein 1989), making use of a diploid potato population and its genetic map, can dissect the underlying genetic factors responsible for the variation observed in this trait. In this paper, we present a combined approach including QTL mapping for ED and the subsequent substrates, candidate gene mapping and expression assays for PPO in order to unravel the complex trait of ED.

\section{Materials and methods}

\section{Plant material}

The diploid potato mapping population $\mathrm{C} \times \mathrm{E}$, descending from the backcross between C (US-W5337.3) (Hannemann et al. 1967) and E (77.2102.37) (Jacobsen 1980), was used. In the current research 249 full sib descendants were selected for which the morphological observations (ED) were done and an AFLP-based linkage map was created (Celis-Gamboa 2002). All clones were grown in multi-year repeats in the field (Wageningen, The Netherlands) during the normal potato-growing season in the Netherlands (April-September).

Measuring the degree of enzymatic discoloration

The field-grown potato tubers were harvested and analyzed directly after harvest. One random tuber per genotype was peeled, grated, and exposed to air in a small Petri dish at room temperature. The degree of discoloration was measured after $30 \mathrm{~min}$ and after $3 \mathrm{~h}$. The measurements for each genotype were done in two replicates and three tubers were tested per replicate. Based on the observed discoloration, the F1 population was visually classified on an ordinal scale in nine different classes (0-8), ranging from no discoloration at all to completely brown/black. The phenotypic analysis was performed in two consecutive years (1998 and 1999).

\section{Measuring tyrosine and chlorogenic acid content}

Tyrosine and chlorogenic acid content were measured in a subset of 100 randomly selected clones of the $\mathrm{C} \times \mathrm{E}$ population. The chlorogenic acid content (levels of 3-, 4-, and 5caffeoyl quinic acid separate) was determined for the harvest of 2002, the tyrosine levels were determined for the harvest of two consecutive years, 2002 and 2003. Directly after harvest $500 \mathrm{~g}$ of tubers were selected per clone and peeled, sliced, and frozen in liquid nitrogen. Then the frozen slices were ground to a very fine powder in liquid nitrogen and stored before analysis at $-80^{\circ} \mathrm{C}$. The analysis of chlorogenic acid levels was done using LCMS-based methods according to (Moco et al. 2006). All samples were measured once and both parent lines were measured six times, in between the progeny samples, to control the stability of the assay. For the tyrosine analysis, tissue $(0.5-1.0 \mathrm{~g})$ was homogenized with a mortar and pestle in $2 \mathrm{ml} 50 \mathrm{mM} \mathrm{Pi}$ buffer (pH 7.0) containing $1 \mathrm{mM}$ dithiothreitol. Nor-leucine was added as an internal standard. Free amino acids were partly purified by extraction with $5 \mathrm{ml}$ of a water : chloroform : methanol mixture $(3: 5: 12)$. The water phase was collected and the organic phase was re-extracted twice. After concentration by lyophilisation to $3 \mathrm{ml}$, a $20-\mu \mathrm{l}$ sample was analyzed by HPLC using a cation-exchange column with post-column ninhydrine derivatisation of the amino acids detected at 570 and $440 \mathrm{~nm}$ (BIOCHROM 20, Amersham Pharmacia biotech, Chalfont, UK).

\section{QTL mapping}

The phenotypic data were tested for normality and, when not normally distributed, transformed (square root). The 
parental maps used in this experiment are obtained from (Celis-Gamboa 2002) with minor modifications. The paternal map consists of 180 dominantly scored AFLP markers grouped together in 12 linkage groups representing the 12 chromosomes. The maternal map contains 141 AFLP markers subsequently grouped together representing the 12 chromosomes. QTL analysis was performed using the software package MapQTL ${ }^{\circledR}$ Version 5.0 (Van Ooijen 2004). Although the phenotypic data consists of values on an ordinal scale, QTL analysis was done using the interval mapping method developed by (Lander and Botstein 1989). The results of the interval mapping were compared to the results of the Kruskal-Wallis test and no differences between the results of the two analyses were observed. Detection of a QTL was done using a LOD threshold calculated with the permutation test option provided in MapQTL (Van Ooijen 2004).

\section{CAPS marker development}

Initially, two forward and reverse primer sets were developed based on mRNA sequence (GB accession number U22921) obtained from NCBI (http://www.ncbi.nlm. nih.gov/). Primers were synthesized by Biolegio BV, Malden, The Netherlands. Primer sets POT32PS1 and POT32PS2 were designed to specifically amplify POT32 sequence through the identification of POT32-specific regions based on alignment of different $\mathrm{PPO}$ sequences [POT32 GB U22921, РОT33 GB U22922, POT72 GB22923, PPO-P1, and PPO-P2 (Hunt et al. 1993)]. POT32PS1 forward primer 5'-CTTCTTCCTCCACTTC TTT-3' and reverse primer GGGCAAAGTTGAACCT-3' and POT32PS 2 forward primer $5^{\prime}$-CCAAAACGTTGAAA CAAA- $3^{\prime}$ and reverse primer $5^{\prime}$-CTCTTGTGCATTTTTC TCT- $3^{\prime}$. PCR reactions were performed using $1 \mu \mathrm{l}$ of DNA $(5 \mu \mathrm{g} / \mu \mathrm{l}), 1.0 \mu \mathrm{l}$ of each primer $(1 \mu \mathrm{g} / \mathrm{ml}), 1 \mu \mathrm{l}$ of dNTPs $(5 \mathrm{mM}), 5 \mu \mathrm{l}$ of PCR buffer, and $0.1 \mu \mathrm{l}$ of Super Taq polymerase (5 U/ml) (Sphaero Q, Leiden, The Netherlands) in a total volume of $20 \mu \mathrm{l}$. The PCR reactions for both sets consisted of a start of $120 \mathrm{~s}$ at $94^{\circ} \mathrm{C}$ and followed by 35 cycles of $94^{\circ} \mathrm{C}$ for $30 \mathrm{~s}, 52^{\circ} \mathrm{C}$ for $30 \mathrm{~s}, 72^{\circ} \mathrm{C}$ for $60 \mathrm{~s}$, and finished by $72^{\circ} \mathrm{C}$ for $300 \mathrm{~s}$. The PCR reactions were performed on PE-9600 thermocycler machines from Perkin-Elmer Applied Biosystems Inc., Foster City, CA, USA. PCR results were analyzed on $1 \%$ agarose gel. PCR fragments were directly cloned into the pGEM $^{\circledR}$-T Easy vector system (Promega Corporation, Madison, WI, USA) using the standard protocol. Plasmid DNA samples, including inserted fragments, were sent to Baseclear BV, Leiden, The Netherlands, for sequencing. Sequences were analyzed using the Vector NTI software package (Informax Inc., Bethesda, MD, USA) The Vector NTI software was also used to identify candidate restriction enzymes with a restriction site polymorphism between the alleles. The predicted restriction site polymorphisms were verified on POT32PS1 PCR fragments amplified from genomic DNA of the parental clones.

\section{Expression analysis}

A selection of genotypes (26 clones) with an extreme phenotype for either strong or weak ED was grown in pots in the greenhouse. Similar sized tubers of a single plant, representing a similar developmental stage having had at least a 4-week period of tuber growth, were selected for expression studies. RNA was isolated as described by (Bachem et al. 1996). Relative Expression levels of POT32 were determined by real-time qRT-PCR on a Perkin-Elmer Abi Prism 7700 Sequence detector (Perkin-Elmer, Nieuwerkerk, The Netherlands) following the protocol described by (Kloosterman et al. 2005). Potato ubiquitin primers (ubi3) were used as a control. Relative quantification of the target RNA expression level and standard deviation was performed using the comparative $\mathrm{Ct}$ method according to the User Bulletin No. 2 (ABI Prism 7700 Sequence Detection System, December 1997, Applied Biosystems, Foster City, CA, USA). The primer sequences for the genes studied are as follows: POT32 (GB accession number U22921) forward primer 5'-AATGCTCCATGTCCTCGGAT-3', reverse primer $5^{\prime}$-AACCCCGAGATCATAAGGCC-3' and $u b i 3$ (GB accession number L22576) forward primer 5'-TTCCGACACCATCGACAATGT-3', reverse primer 5'-CGACCATCCTCAAGCTGCTT-3'.

\section{Results}

Phenotypic analysis

Enzymatic discoloration values of the population represent the means of all replicates, over years and repetitions. The values showed a large variation, ranging from 0 to 8 , several clones showed no discoloration at all, even after $3 \mathrm{~h}$, while other genotypes developed severe discoloration (Fig. 1a). ED shows a transgressive segregation pattern in which both parental clones have intermediate phenotypes. The broad sense heritability's were estimated as 0.84 for discoloration after 30 min and 0.82 for discoloration after $3 \mathrm{~h}$.

Since tyrosine and chlorogenic acid (as the main polyphenolic compound) are the principal substrates for enzymatic browning, the levels of these compounds were determined for each potato clone. The results of these measurements are shown as averages over 2 years per clone for tyrosine in Fig. 1b and for chlorogenic acid as squareroot transformed values (to compensate for the absence of normal distribution) of 1 year in Fig. 1c. The smallest 
Fig. 1 Distribution of the trait values in the $\mathrm{C} \times \mathrm{E}$ offspring. Letters $\mathrm{C}$ and $\mathrm{E}$ above the graphs indicate parental values. a Degree of enzymatic discoloration on a scale of 0 (no discoloration) to 8 (dark brown/black). b Tyrosine content ( $\mu \mathrm{mol}$ per gram tuber flesh). c Chlorogenic acid (relative scale)
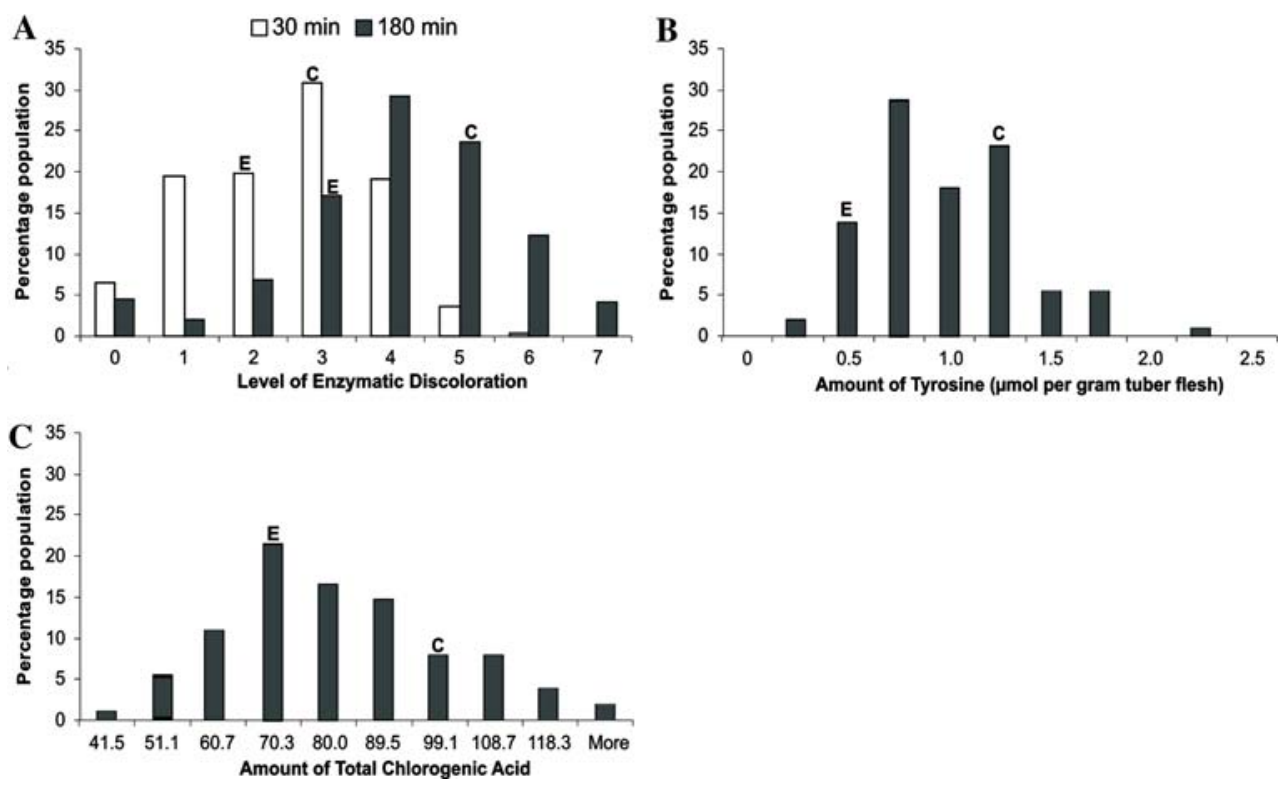

amount of tyrosine present was $0.232 \mu \mathrm{mol}$ per gram fresh tuber flesh and the highest amount was $2.143 \mu \mathrm{mol}$ per gram, a more than ninefold increase in tyrosine content between the two genotypes. For this trait the heritability was estimated to be 0.88 . The three different chlorogenic acid forms (3-, 4-, and 5-caffeoyl quinic acid) investigated, showed a variation of 10-50-fold changes in amount between clones. The correlation coefficient between the degree of ED and tyrosine levels was 0.20 (with $P=0.05$ ) and between enzymatic browning and chlorogenic acid levels the correlation was 0.13 (with $P=0.20$ ). Based on the data obtained from this population, we can conclude that there appears to be no relationship between assayed substrate levels and the degree of ED.

\section{QTL mapping}

In order to identify the chromosomal regions contributing to the phenotypes of ED, tyrosine levels and chlorogenic acid levels, QTL analyses were performed. QTLs were identified based on a genomewide significant threshold obtained by performing a permutation test on the data set. For both time points at which discoloration was determined, QTLs were identified on both the $\mathrm{C}$ and $\mathrm{E}$ parental maps. The QTLs of the different time points coincide precisely. However, since the QTL effects at the $30 \mathrm{~min}$ time point are significantly larger than those at $3 \mathrm{~h}$, which do not all reach the LOD threshold, only the QTLs for the $30 \mathrm{~min}$ time point are shown in Fig. 2a.

In total, four QTLs were identified affecting the degree of ED, two on the maternal map (ED-3 on chromosome C3 and ED-8a on C8) and two on the E map (ED-1 on chromosome E1 and ED-8b on E8). The QTLs on chromosome 8 of both the parental maps are homologous to each other, both co-localizing with the markers for POT32. QTL ED8a explains the largest part $(21 \%)$ of the total variance for this trait. The QTLs on C3, E1 and E8 have explained variances of 20,12 , and $16 \%$, respectively, although both QTLs on chromosome 8 explain, partly, the same variance twice. The candidate gene POT32 (see below), specifically allele 1 , shows the highest association with the QTL on chromosomes 8 of both the parental maps in the non-parametric Kruskal-Wallis test $\left(K^{*}=33,227\right.$ with $1 d f$ and $P<0.0001)$.

QTL analysis for chlorogenic acid content was performed on all the three forms (3-, 4-, 5-, and caffeoyl quinic acid) independently and on total chlorogenic acid content. Only the analysis for 5-caffeoyl quinic acid gave a significant result above the LOD threshold. One single QTL (CA2) was detected on chromosome $\mathrm{C} 2$ (Fig. 2b). The QTL has a LOD score of 3.80 and explains $21 \%$ of the total variance. For the other substrate, tyrosine, and QTL analysis was performed using the averaged data over the two harvest years. The analysis resulted in a single significant QTL at chromosome C8, Tyr-8 (Fig. 2c), with a LOD score of 5.76 and an explained variance of $36 \%$.

As can be seen from Fig. 2, the QTLs for ED and for both the substrates do not co-localize. Both ED and tyrosine content do show a QTL on chromosome C8. However, the QTLs are more than $20 \mathrm{cM}$ apart, without an overlap in the QTL intervals, thus indicating that both QTLs are independant. Although the QTL analysis of ED results in three different QTLs and the substrate-based components result in two QTLs, none of these QTLs overlap. Contrary to our expectations we cannot prove a clear correlation between ED and substrate levels. 
Fig. 2 a LOD profiles of the interval mapping analysis for the trait enzymatic discoloration. Shown are the observed QTLs on chromosomes $\mathrm{C} 3, \mathrm{C} 8, \mathrm{E} 1$, and E8. b The LOD profiles of the interval mapping analysis for the chlorogenic acid (5-caffeoyl quinic acid) content, showing the observed QTL on chromosome C2. $\mathbf{c}$ The LOD profiles of the interval mapping analysis for tyrosine content, showing the QTL on chromosome C8. The LOD thresholds in all figures have been set at 3.0

\section{A $\quad$ C3}
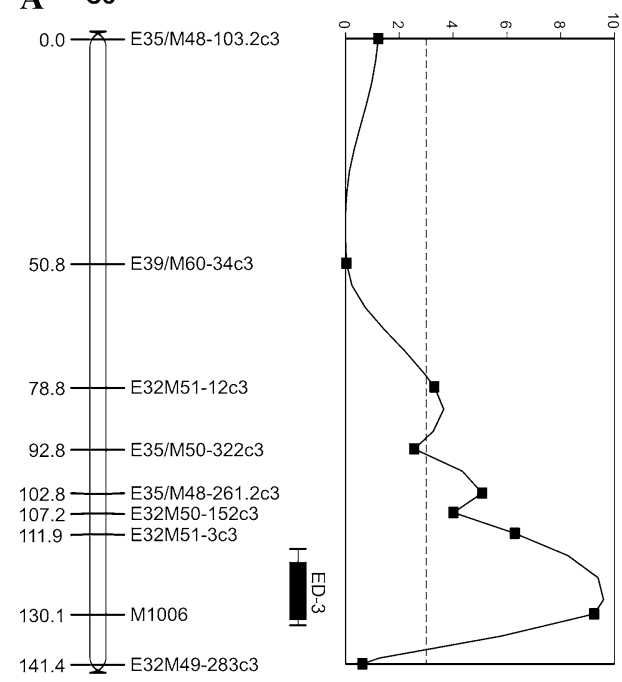

E1
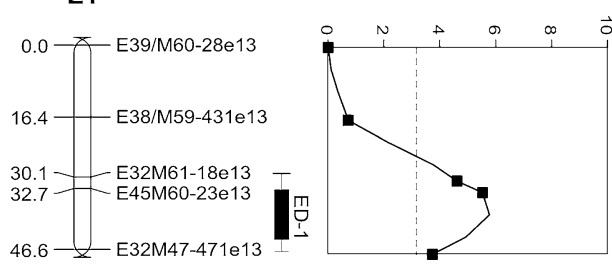

B C2

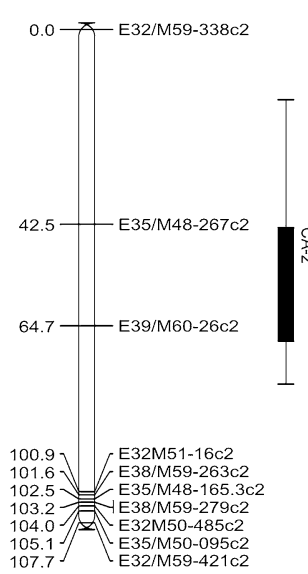

C8

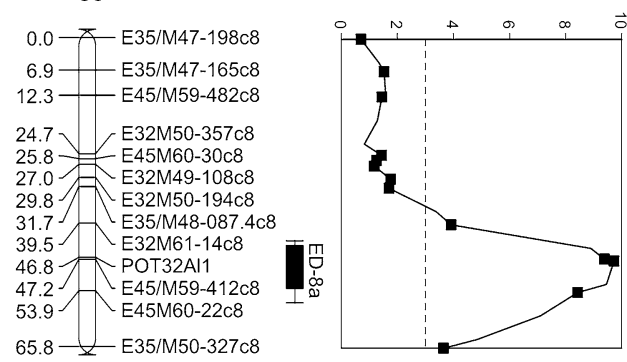

$0.0]$ E8 E32/M48-323.7e8

$4.1]$ E8 [ E32M61-27e8

6.7 ] E32M51-8e8

$8.0]=232 \mathrm{M} 51-11 \mathrm{e} 8$

8.3 E32M51-35e8

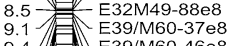

$\left.\begin{array}{r}9.4 \\ 10.2\end{array}\right]\left[\begin{array}{l}1.1 \\ E 39 / M 60-46 e 8 \\ E 38 / M 59-333 e 8\end{array}\right.$

11.8
12.
$E 32 M 51-31 \mathrm{e} 8$

12.0
12.6 $\left.\begin{array}{l}13.7 \\ 16.0\end{array}\right] \begin{aligned} & E 32 M 54-73 \mathrm{e} 8 \\ & E 32 \mathrm{M} 54-90 \mathrm{e} 8\end{aligned}$ $\left.\begin{array}{l}16.0 \\ 17.8\end{array}\right]$ E32M54-90e8 $\left.\begin{array}{l}40.3 \\ 41.4\end{array}\right]\left[\begin{array}{l}\text { E } \\ \text { POT32Al3 } \\ \text { POT32Al1 }\end{array}\right.$

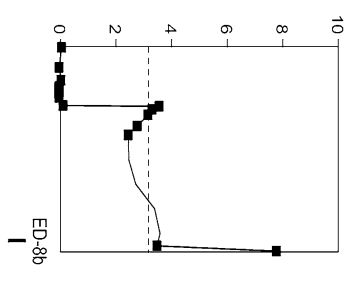

C $\quad$ C8

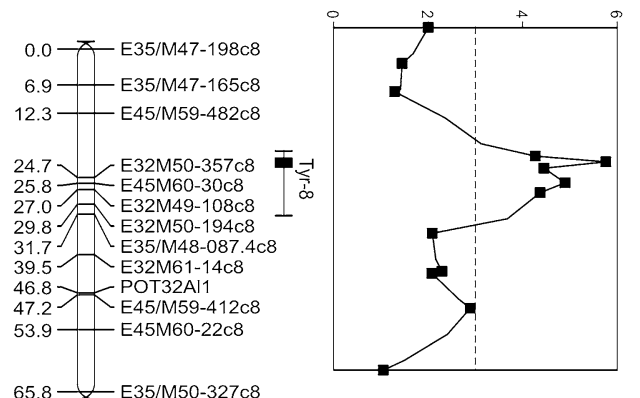

$65.8-$ E35/M50-327c8
Candidate gene allele identification and mapping

The major candidate gene for ED is PPO. From all the members of this gene family POT32 and POT33 show tuber expression. Although the POT33 gene shows a low expression in tubers, POT32 is likely to be responsible for discoloration as it is the most abundantly expressed member of the PPO gene family in tubers. Therefore, POT32 was chosen for further analysis. In order to verify POT32 specificity and to display the available allelic diversity in this population, two PCR primer pairs were designed on overlapping sections of the POT32 gene. The PCR products were cloned, sequenced and the sequences were sub-

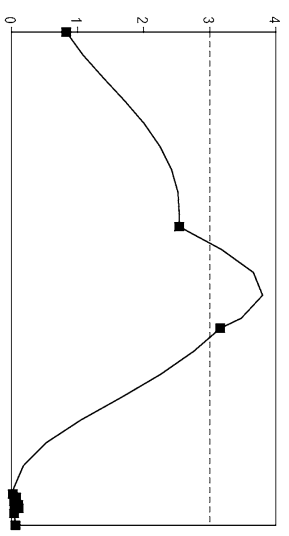

sequently compared to the available PPO sequences. A single primer pair was shown to be POT32-specific (POT32PS1; giving a 990 bp fragment). Sequence from the other primer pair (POT32PS2; giving a 1,300 bp fragment) showed amplification of POT32, POT33, and POT72 genes. Sequence from the POT32 gene obtained with POT32PS2 primer pair allowed the identification of allelic variation within the $\mathrm{Cu}$ binding domain $\mathrm{B}$ as described below.

Three distinct allelic versions of POT32 were observed in the parental genotypes and designated alleles 1, 2, and 3 . Allele 1 is shared between both parents. An alignment of the predicted protein sequence of each of the alleles and of 
a reference sequence, U22921, is shown in Fig. 3. Parent C harbors alleles 1 and 2 and parent $E$ has alleles 1 and 3 . Allele 1, which is identical to the published sequence of POT32 (GB accession number U22921), has 97\% homology with allele 2 on the nucleotide level and $98 \%$ homology with allele 3 , alleles 2 , and 3 are $98 \%$ identical. In the 990 bp sequence, 25 SNPs occur when comparing allele 2 with allele 1 , the 25 SNPs give rise to 15 amino acid changes. For allele 3, 29 SNPs in 1,425 bp sequence are observed that result in 12 amino acid changes. As indicated in the alignment in Fig. 3, some of these amino acid changes occur in the conserved $\mathrm{Cu}$-binding domains $\mathrm{A}$ and $\mathrm{B}$. One of the changes in allele 2 is in the third histidine of $\mathrm{Cu}$-binding domain $\mathrm{A}$. The histidines in the $\mathrm{Cu}$-domains are very well conserved throughout plant species (Marusek et al. 2006). Amino acid changes in this region is likely have an effect on the activity of the enzyme.

Restriction enzyme digestion of the PCR fragments with the enzyme $H p h \mathrm{I}$, enables the identification of allele 3 due to the absence of an $H p h \mathrm{I}$ restriction site at base pair 733 . Allele 1 can be identified using enzyme HpyF10IV, as the allele 1 sequence harbors an extra $H p y F 10 I V$ restriction site at residue 638. In this way CAPS markers for alleles 1 and 3 of POT32 were developed and the segregation was analyzed for the complete $\mathrm{C} \times \mathrm{E}$ population and used to map POT32 on the genetic linkage map of $\mathrm{C} \times \mathrm{E}$. POT32 alleles 1 and 3 map together on chromosome E8. Allele 1 is present in both parents and as such also maps on chromosome $\mathrm{C} 8$.

The effect of the different alleles, on the degree of ED, can be indicated by grouping all the genotypes of the population according to their allelic composition for POT32 and calculating the mean of the ED scores. An overview of this grouping is given in Fig. 4. In the $\mathrm{C} \times \mathrm{E}$ population four different allele compositions can be identified (homozygous for allele 1 , allele 1 plus allele 2 , allele 1 plus allele 3 , and the combination of allele 2 with allele 3 ). For both time points at which discoloration was measured, the group of clones homozygous for allele 1 showed more severe discoloration. On the other hand, the group of clones carrying alleles 2 and 3 displayed significantly less ED. The other two-allele combinations show intermediate phenotypes.

PPO expression analysis, using qRT-PCR, was performed on a subset of extreme clones to investigate the

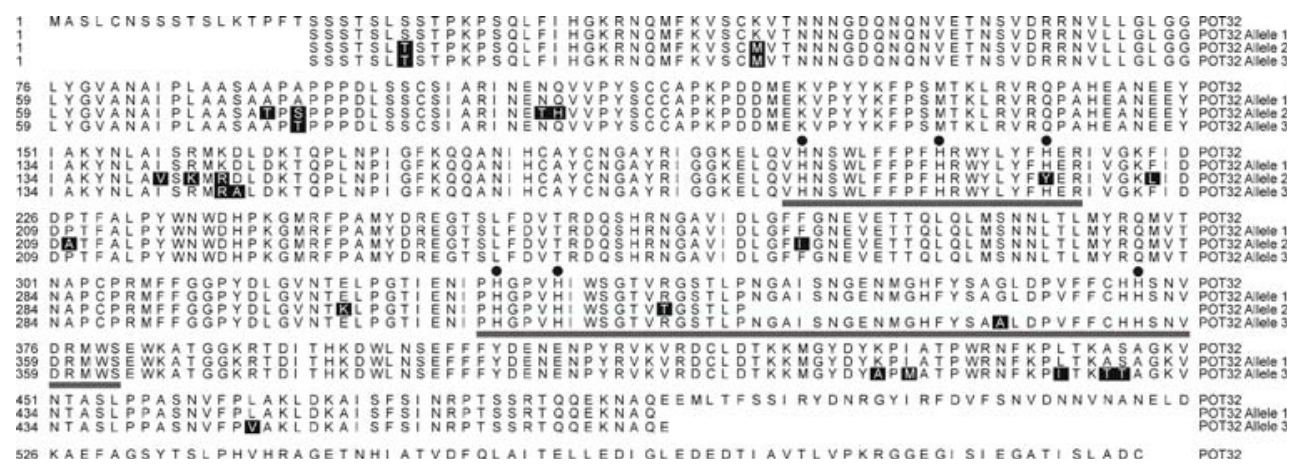

Fig. 3 Alignment of POT32 allele protein sequences with POT32 STU22922 from database. Indicated in black are polymorphisms compared to POT32 STU22922. Indicated with black underlining are the

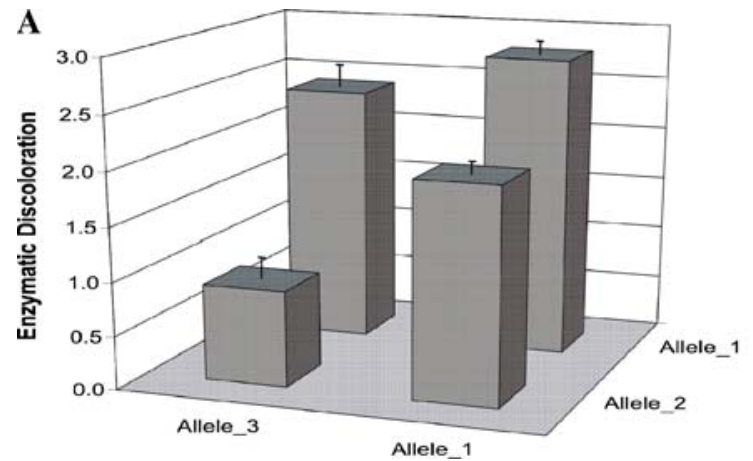

Fig. 4 The means of the discoloration scores per allele combination, based on the data of the complete $\mathrm{C} \times \mathrm{E}$ population. The error bars represent the standard error of the means of each allelic combination. The class of allelic combination $1+1$ comprises 55 genotypes, combi- two Cu-binding domains A and B (Thygesen et al. 1995), and with the black dots above the alignment the histidines in these $\mathrm{Cu}$-binding domains are indicated

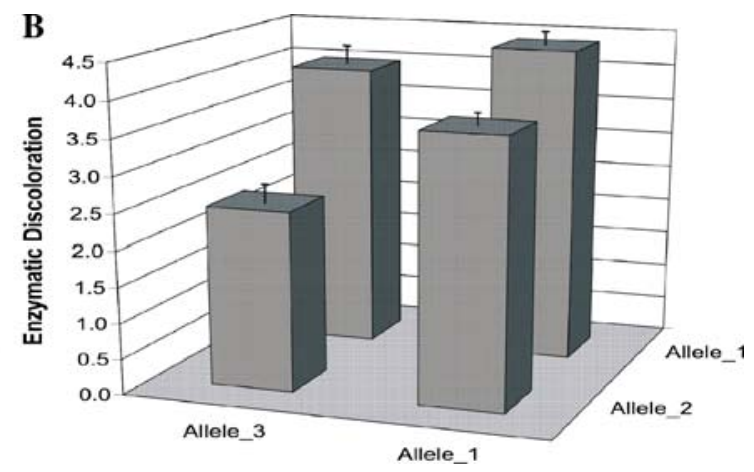

nation $1+2$ contains 61 genotypes, and combinations $1+3$ and $2+3$ contain 38 and 30 genotypes, respectively. a Represents the scores at time point $30 \mathrm{~min}$. b Shows the scores at time point $3 \mathrm{~h}$ after grading of the potato tuber flesh 
possibility of allele-dependent differential expression. Due to the high-sequence similarity between the individual alleles, we were not able to design allele-specific primers. POT32-gene specific primers were therefore designed in regions for which all three alleles were identical to allow assessment of the sum of transcripts. By using the four different offspring genotypes, the expression levels for allele combinations could be estimated. Figure 5 indicates that the clones homozygous for allele 1 have, on average, the highest expression levels and the group with a combination of alleles 2 and 3 has on average the lowest levels of expression. This result shows consistency with the previous results for the ED scores. On the basis of the combined results for the phenotypes and the expression levels, it can be deduced that in this population, РОT32 allele 1 appears to have the highest expression level and its presence relates to a discoloring phenotype. In general, we conclude that variation in discoloration appears to correlate to expression level variation as well as variation in the allelic composition.

\section{Discussion}

In this paper, we present the genetic architecture underlying the ED of potato tubers caused by the formation of dark melanin pigments through the oxidation of phenolic compounds by the enzyme PPO. Although it is the first time this trait is analyzed by means of QTL analysis and a candidate gene approach, extensive research has been performed on the trait focussing on both the enzymatic components (Hunt et al. 1993; Newman et al. 1993; Thygesen et al. 1995) and substrate components (Corsini et al. 1992; Marri et al. 2003; Mondy and Munshi 1993). The enzymatic component is represented by the enzyme PPO. To explain the sub-

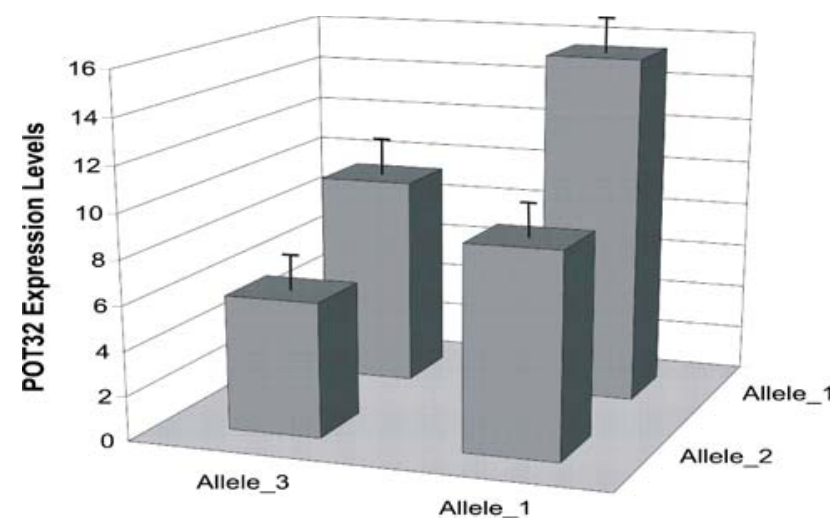

Fig. 5 The means of the expression levels for POT32 per allele combination in a selection of extreme phenotype clones for enzymatic discoloration. The error bars represent the standard error of the means of each allelic combination. The classes of allelic combination $1+1$, $1+2,1+3$, and $2+3$ contain $6,6,7$, and 7 genotypes, respectively. The expression levels are expressed as fold difference relative to the lowest expressing clone strate components involved in discoloration the levels of two substrates of PPO have been examined, namely tyrosine and chlorogenic acid.

The most obvious candidate gene for ED is PPO, more specifically the gene POT32. This gene is primarily only expressed in tuber and roots and has the highest expression of the tuber expressed PPO genes (Thygesen et al. 1995). The function of the enzyme in the enzymatic reaction has been well established and is mapped at chromosome 8 of the potato genome (Tanksley et al. 1992). This map position was confirmed by mapping PPO gene POT32 present in the $\mathrm{C} \times \mathrm{E}$ population at chromosome 8 , as can be observed from the map of C8 and E8 in Fig. 2a.

Three different POT32 alleles in this population could be identified, with 97-98\% homology at the nucleotide level. When translated to the protein sequence, the different allelic forms still share between 95 and $98 \%$ homology. A number of amino acid changes also occur in the active Copper-Binding domains, raising the possibility of an alteration in the activity of the enzyme. Figure 4 indicates that the combination of the alleles 2 and 3 , or the absence of allele 1, results in a lower degree of ED. This result is visible at both time points of measuring ED ( $30 \mathrm{~min}$ and $3 \mathrm{~h}$ ). Interestingly, the results of the POT32 expression analysis are in agreement with the results shown in Fig. 4. Genotypes with the combination of alleles 2 and 3, conferring low ED, seem to exhibit, lower average levels of POT32 expression. This result indicates that, the differential PPO-gene expression may also be a factor in the observed phenotypes. SNPs in CIS-acting elements of the POT32 gene, are likely to result in the different observed levels of expression of the different alleles. However, we cannot exclude that these differences in expression levels merely reflect differences in available substrate levels or other genotype $\times$ environment interactions.

The QTL analysis of ED has revealed the contribution of four QTLs on chromosomes 3 and 8 of the maternal (C) and chromosome 1 and 8 of the paternal (E) map to the trait ED. While we have elucidated one of the genetic factors underlying the QTLs for ED, namely POT32 and the QTL on chromosome 8, two genes underlying other QTLs, ED-1, and ED-3, remain to be identified. ED-3 possibly co-localizes with the $Y$-locus mapped in potato on chromosome 3 (Bonierbale et al. 1988). The $Y$-locus controls tuber flesh color and likely candidate genes for this locus are phytoene synthase and/or $\beta$-carotene hydroxylase (Thorup et al. 2000). A possible function of these genes, in relation to ED, can be deduced from results obtained in apricot (Rigal et al. 2003), where it was observed that ED could be inhibited by trans- $\beta$-carotene, either by direct inhibition of PPO or by phenol regeneration through non-enzymatic reactions with quinones. Both genes are involved in the carotenoid biosynthesis pathway. For the QTL ED-1, possible candidates genes can be involved in the production of enzymatic 
browning inhibitors such as ascorbic acid (Matheis and Belitz 1977) or thiol compounds such as cysteine (Richard et al. 1991). Besides inhibitors of discoloration, genes involved in the production of phenolic compounds other than tyrosine or chlorogenic acid, may also play a role in the process of ED such as cinnamic or caffeic acids (Friedman 1997). OTL analysis of the chlorogenic acid and tyrosine levels resulted in QTLs CA-2 and Tyr-8, respectively. Previous reports indicate a correlation between phenolic content and ED (Corsini et al. 1992; Mondy and Munshi 1993). In our population this correlation could not be observed.

The identification of three QTLs within the potato genome influencing the trait of ED in potato tubers represents a substantial contribution to the understanding of this complex trait. QTL ED-8a can be explained by the PPO gene POT32, the possible relation of ED-3 with the $Y$-locus could clarify the genetics, underlying this trait, further. The correlation with the substrates tyrosine and chlorogenic acid as described by other authors, could not be confirmed in our experiments. Although the selection for PPO enzyme activity may not be an effective strategy for breeding (Brown et al. 1994), the direct selection for the different alleles of POT32 may help in achieving non-discoloring cultivars. Knowledge on the different allelic forms of POT32 and the effect of their presence or absence in the potato genome is a very promising result from a breeding point of view.

Acknowledgments This project was financed by the Centre for BioSystems Genomics (CBSG) which is part of the Netherlands Genomics Initiative/Netherlands Organisation for Scientific Research. We are grateful to Herman van Eck (Laboratory of Plant Breeding, Wageningen University and Research Centre, Wageningen, The Netherlands) for his contribution to the fruitful discussions on the topics of this article.

\section{References}

Bachem CW, van der Hoeven RS, de Bruijn SM, Vreugdenhil D, Zabeau M, Visser RG (1996) Visualization of differential gene expression using a novel method of RNA fingerprinting based on AFLP: analysis of gene expression during potato tuber development. Plant J 9:745-753

Bachem CWB, Speckmann GJ, van der Linde PCG, Verhaggen FTM, Hunt MD, Zabeau M (1994) Antisense expression of polyphenoloxidase genes inhibits enzymatic browning of potato tubers. Biotechnology 12:1101-1127

Bonierbale MW, Plaisted RL, Tanksley SD (1988) RFLP maps based on a common set of clones reveal modes of chromosomal evolution in potato and tomato. Genetics 120:1095-1103

Brown C, McNabnay M, Dean B (1994) Genetic characterization of reduced melanin formation in tuber tissue of Solanum hjertingii and hybrids with cultivated diploids. Am J Potato Res 76:37-43

Celis-Gamboa BC (2002) Life cycle of the potato (Solanum tuberosum L.): from crop physiology to genetics. Ph.D. Thesis, Wageningen University, Wageningen, The Netherlands

Corsini DL, Pavek JJ, Dean B (1992) Differences in free and proteinbound tyrosine among potato genotypes and the relationship to internal blackspot resistance. Am Potato J 69:423-435
Culley D, Yang C-P, Dean B, Brown C (2000) Characterization of the polyphenol oxidase gene family from the low browning Solanum species, S. hjertingii. In: Abstracts presented at the 84th annual meeting of the potato association of America, Colorado Springs, Colorado, USA

Friedman M (1997) Chemistry, biochemistry, and dietary role of potato polyphenols. A review. J Agric Food Chem 45:1523-1540

Hannemann RE, Peloquin SJ (1967) Crossability of 24-chromosome potato hybrids with 48-chromosome cultivars. Eur Potato J 10:62-73

Hunt MD, Eannetta NT, Yu H, Newman SM, Steffens JC (1993) cDNA cloning and expression of potato polyphenol oxidase. Plant $\mathrm{Mol}$ Biol 21:59-68

Jacobsen $E$ (1980) Increase of diplandroid formation and seed set in $4 x$ $\mathrm{X} 2 \mathrm{x}$ crosses in potatoes by genetical manipulation of diphaploids and some theoretical consequences. Z Planzenzuecht 85:110-121

Kloosterman B, Vorst O, Hall RD, Visser RGF, Bachem CWB (2005) Tuber on a chip: differential gene expression during potato tuber development. Plant Biotechnol J 3:505-519

Lander ES, Botstein D (1989) Mapping mendelian factors underlying quantitative traits using RFLP linkage maps. Genetics 121:185-199

Marri C, Frazzoli A, Hochkoeppler A, Poggi V (2003) Purification of a polyphenol oxidase isoform from potato (Solanum tuberosum) tubers. Phytochemistry 63:745-752

Marusek CM, Trobaugh NM, Flurkey WH, Inlow JK (2006) Comparative analysis of polyphenol oxidase from plant and fungal species. J Inorg Biochem 100:108-123

Matheis G, Belitz HD (1977) Studies on enzymatic browning of potatoes (Solanum tuberosum). II. The quantitative relationship between browning and its causative factors. Z Lebensm Unters Forsch 163:186

Moco S, Bino RJ, Vorst O, Verhoeven HA, de Groot J, van Beek TA, Vervoort J, de Vos CH (2006) A liquid chromatography-mass spectrometry-based metabolome database for tomato. Plant Physiol 141:1205-1218

Mondy NI, Munshi CB (1993) Effect of maturity and storage on ascorbic acid and tyrosine concentrations and enzymatic discoloration of potatoes. J Agric Food Chem 41:1868-1871

Newman SM, Eannetta NT, Yu H, Prince JP, de Vicente MC, Tanksley SD, Steffens JC (1993) Organisation of the tomato polyphenol oxidase gene family. Plant Mol Biol 21:1035-1051

Richard FC, Goupy PM, Nicolas JJ, Lacombe JM, Pavia AA (1991) Cysteine as an inhibitor of enzymatic browning. 1. Isolation and characterization of addition-compounds formed during oxidation of phenolics by apple polyphenol oxidase. J Agric Food Chem 39:841-847

Rigal Dd, Gauillard F, Richard-Forget F (2003) Changes in the carotenoid content of apricot (Prunus armeniaca, var Bergeron) during enzymatic browning: $\beta$-carotene inhibition of chlorogenic acid degradation. J Sci Food Agric 80:763-768

Tanksley SD, Ganal MW, Prince JP, de Vicente MC, Bonierbale MW, Broun P, Fulton TM, Giovannoni JJ, Grandillo S, Martin GB, et al (1992) High density molecular linkage maps of the tomato and potato genomes. Genetics 132:1141-1160

Thorup TA, Tanyolac B, Livingstone KD, Popovsky S, Paran I, Jahn M (2000) Candidate gene analysis of organ pigmentation loci in the Solanaceae. Proc Natl Acad Sci USA 97:11192-11197

Thygesen PW, Dry IB, Robinson SP (1995) Polyphenol oxidase in potato. A multigene family that exhibits differential expression patterns. Plant Physiol 109:525-531

Van Ooijen JW (2004) MapQTL ${ }^{\circledR} 5$, Software for the mapping of quantitative trait loci in experimental populations. In: Kayzma BV (ed) Wageningen, Netherlands

Vaughn KC, Lax AR, Duke SO (1988) Polyphenol oxidase-the chloroplast oxidase with No established function. Physiol Plant 72:659-665 